\title{
Reduction of the Pathways of Photochemical Gasphase Reactions Induced by Multiple Photon IR Laser Excitation of Molecules
}

\author{
V. N. BAGRATASHVILI, M. V. KUZMIN and V. S. LETOKHOV
}

(Received 15 June 1982; in final form 25 April 1983)

Two different cases, (1) thermal initiation and (2) collisionless IR multiple photon (MP) initiation, of initiating the model reaction of the successive decay of polyatomic molecules $A B C \rightarrow A B+C, A B \rightarrow A+B$ are considered for the case when the intermediate product $A B$ is desired. In the case when the desired product $A B$ is thermally less stable than the original one $A B C$ the high output of $A B$ under the thermal initiation is impossible. However by means of IR MP initiation one can obtain a high yield of the desired product, i.e., nearly $100 \%$. The reduction of the chemical reaction pathways takes place for the IR MP initiation. This is due to the extreme intermolecular nonequilibrium achieved under IR MP initiation of the reaction. Unlike the thermal initiation when all the reaction products are of equally high temperature, under IR MP initiation the temperature of the intermediate product $T_{A B}$ is lower than that of the original one $T_{A B C}$ and a further decay of the $A B$ species does not occur.

\section{INTRODUCTION}

It is well known that during thermal initiation of gas-phase radical chemical reactions of polyatomic molecules, a great number of final products are usually formed and the yield of the desirable product is often rather low. This rather common situation is conditioned by a great number of potential chemical reactions in multicomponent mixtures, when under thermodynamic equilibrium the initial, intermediate and final products have the same high temperature.

Of considerable interest in this respect are chemical reactions under nonequilibrium and nonstationary conditions, when the temperatures 
of the initial, intermediate and final products are quite different. Photochemical reactions may fall in this category. As known, photochemical reactions taking place as the electronic states of molecules are excited, essentially differ from thermal reactions (see, for example, Ref. 1).

Recent years have seen one more possibility of performing photochemical reactions in gas-phase through multiphoton excitation of the high vibrational levels of a polyatomic molecule by a powerful pulse of IR laser radiation. ${ }^{2-5}$ In some works successful experiments have been performed on synthesis of molecular compounds under the conditions of a gas-phase radical reaction during IR multiphoton excitation of one of the initial molecular compounds. In Ref. 6, for example, effective synthesis of the $\left(\mathrm{CF}_{3}\right)_{3} \mathrm{CI}$ molecule during IR photodissociation of $\left(\mathrm{CF}_{3}\right)_{3} \mathrm{CBr}$ in the presence of $\mathrm{I}_{2}$ was observed. In this experiment one could observe controllable direct synthesis of the $\left(\mathrm{CF}_{3}\right)_{3} \mathrm{CI}$ molecule with a high yield (up to $60 \%$ ), and the formation of other products typical of thermal reactions was suppressed. In qualitative respect this is quite understandable since in IR photochemical dissociation of original molecules (as well as in UV photochemistry) the resulting radical-products have comparatively low energy.

The purpose of this paper is to give a more general explanation to a high degree of directivity, that is, reduction of the number of path-ways of the photochemical reaction stimulated by the MP excitation of the molecule. Using a simple model we are going to show that, in the process of IR MP photodissociation, it is possible to obtain the only desirable reaction product with a yield close to the limiting one, i.e., $100 \%$.

\section{PHOTOCHEMICAL REACTION MODEL}

Here we are going to restrict ourselves to considering the case of successive dissociation of a polyatomic molecule $A B C$ according to the scheme

$$
\begin{gathered}
A B C \stackrel{k_{1}}{\longrightarrow} A B+C \\
A B \stackrel{k_{2}}{\longrightarrow} A+B
\end{gathered}
$$


We shall not discriminate between the cases when the products $A B$, $C, A$ and $B$ are molecules or radicals. In the last case it is assumed that the reaction takes place with a sufficient amount of radical scavenger, so that all the resultant radicals are bound into stable final products.

To demonstrate a high degree of directivity of laser-induced reactions let us consider the case when the thermal rate of reaction (2) is higher than the rate of reaction (1), i.e.,

$$
k_{2} \gg k_{1}
$$

and $A B$ is the desirable product. It is clear that in this case it is impossible to obtain a high yield of $A B$ at thermal initiation since, according to (3), $A B$ dissociates even faster than it is formed. We are going to show that, if reactions (1) and (2) take place under the conditions of $M P$ collisionless photodissociation, the yield of $A B$, despite (3), may be as high as $100 \%$. The resulting $A B$ molecules are cold, because their average energy is less, by at least the value of energy of the $A B-C$ bond, than that of dissociating $A B C$ molecules. Their further decay (2) is possible only after absorbing some energy comparable to the energy of breaking of the $A-B$ bond $D_{2}$. Therefore, if the rate of $M P$ excitation is properly chosen, dissociation (2) does not occur and the desirable $A B$ product is produced. But when the reaction proceeds under thermal equilibrium, the resulting $A B$ molecules get quickly heated in collisions with the hot initial substance $A B C$ and so dissociate at a very high rate.

\section{THERMAL INITIATION OF REACTION}

Let us consider the case of reaction under thermal equilibrium. The kinetic equations for the reaction of successive dissociation (1) and (2) can be presented in the form: ${ }^{7}$

$$
\begin{aligned}
\frac{d \beta}{d t} & =k_{1}(1-\beta) \\
\frac{d \beta_{A B}}{d t} & =k_{1}(1-\beta)-k_{2} \beta_{A B}
\end{aligned}
$$

where $\beta$ and $\beta_{A B}$ are respectively the primary yield of dissociation reaction (1) and the yield of the desirable $A B$ product in the presence 
of reaction (2), i.e.,

$$
\begin{aligned}
\beta & =1-\frac{[A B C]}{[A B C]_{0}}=\frac{[A B]+[A]}{[A B C]_{0}} \\
\beta_{A B} & =\frac{[A B]}{[A B C]_{0}}
\end{aligned}
$$

Let the solution of system (4) be presented as the dependence of the relative yield of the desirable $A B$ product $\beta_{A B} / \beta=$ $[A B] /([A B]+[A])$ on the total yield of $A B C$ dissociation. It is the value $\beta_{A B} / \beta$ that reflects the degree of directivity of the chemical reaction. With $k_{2} \neq k_{1}$ solution (4) is expressed by the formula

$$
\frac{\beta_{A B}}{\beta}=\frac{1}{k_{2} / k_{1}-1} \frac{1-\beta}{\beta}\left[1-(1-\beta)^{k_{2} / k_{1}-1}\right]
$$

With $k_{2} / k_{1} \gg 1$ a high relative yield of the desirable product is possible only at low degrees of processing of the initial product $\beta \ll 1$. At the same time for considerable values of $\beta$ the value of $\beta_{A B} / \beta$ is very small. Specifically, with $\beta=\frac{1}{2}$ and $k_{2} / k_{1} \ll 1$ expression (6) changes to

$$
\left(\frac{\beta_{A B}}{\beta}\right)_{1 / 2}=\frac{1}{k_{2} / k_{1}-1} \ll 1
$$

Thus, at thermal initiation of reaction it is impossible to carry on effective synthesis of the desirable $A B$ product.

It must be noted that, in order to apply expression (6), it is necessary and sufficient that the ratio $k_{2} / k_{1}$ should be constant in the course of the reaction. The area of its applicability, however, is not limited by reactions performed in a thermostat. Even when the medium is heated by pulsed IR radiation, it turns out ${ }^{8}$ that the basic part of the reaction takes place under temperature stabilization when its variation, as $\beta$ increases for example from $0.1-0.9$, is very small. Besides, the variation of $k_{2} / k_{1}$ can be neglected even though the temperature changes greatly but the activation energies of reactions (1) and (2) are close to each other.

\section{IR MULTIPHOTON INITIATION OF REACTION}

Here we consider the case when reactions (1) and (2) are initiated by IR MP absorption by $A B C$ and $A B$ molecules. In this case it is 
possible to realize effective formation of the desirable $A B$ product with $\beta_{A B} / \beta \approx 1$ at high yields $\beta \approx 1$, even despite condition (3). To find the values $\beta$ and $\beta_{A B}$ it is necessary that we should know the distribution over vibrational states formed during MP excitation $f(E)$ ( $E$ is the vibrational energy of molecule). To derive the function $f(E)$ one should solve the systems of appropriate kinetic equations describing energy absorption from the laser field and dissociation (see, for example, Refs. 3 and 4). As a matter of fact, they cannot be solved in the general form and so numerical computation is usually used in correlation with experiment. In certain approximations, however, it is possible to obtain rather simple analytical solutions of these equations. For instance, in an approximation of an $s$-multiple degenerate linear oscillator at the levels where dissociation does not play a significant role the distribution of vibrational energy of a polyatomic molecule turns out to be a Boltzmann function ${ }^{9,10}$

$$
f_{n}=g_{n}\left[\exp \left(-\frac{n \hbar \omega}{k T}\right)\right] / z
$$

where $g_{n}=(n+s-1) ! / n !(s-1) !$ is the multiplicity of degeneration of the $n$-th level, and $z$ denotes the vibrational statistical sum. The temperature $T$ depends on the value of stored energy $\bar{E}$ (or the number of stored IR quanta $\bar{n}$ ).

$$
\bar{n}=\frac{\bar{E}}{\hbar \omega}=\frac{s}{\exp (\hbar \omega / k T)-1}
$$

where $\omega$ is the vibrational quantum frequency. For distribution (7) we can easily obtain the value of dispersion, that is, the energy distribution value $f_{n}(E)$

$$
\delta E=\hbar \omega \delta n=\left(\hbar \omega \bar{E}+\bar{E}^{2} / s\right)^{1 / 2}
$$

With $\bar{n} \gg 1$ and $s \gg 1$ distribution (7) is narrow enough, that is, $\delta n / \bar{n}=$ $\sqrt{1 / s+1 / \bar{n}} \ll 1$. It should be noted that the calculations ${ }^{4}$ of the excitation dynamics for more realistic models result in narrower distributions. To estimate the values of dissociation yield $\beta$ and relative yield of desirable product $\beta_{A B} / \beta$ we are going to use relation (9) thus overestimating the distribution $f(E)$ width. Even though $f(E)$ inevitably causes the value of $\beta_{A B} / \beta$ to be underestimated, we shall see that the values of $\beta_{A B} / \beta$ obtained will be rather high. The whole 
ensemble of the $A B C, A B$ and $A$ molecules that absorb radiation will be described with the composite function $F_{\mathrm{MP}}(E)$ depending on the total energy $E$ which comprises not only the vibrational energy but also the energy of breaking of bonds $D_{1}$ and $D_{2}$

$$
F_{\mathrm{MP}}(E)=f^{A B C}(E)+f^{A B}\left(E-D_{1}\right)+f^{A}\left(E-D_{1}-D_{2}\right)
$$

The functions $f^{A B C}(E), f^{A B}(E)$ and $F^{A}(E)$ describe the vibrational energy distribution in $A B C, A B$ and $A$ respectively. Definition (10) comprising the shifts of initial vibrational energy by $D_{1}$ for $A B$ and by $D_{1}+D_{2}$ for $A$ is convenient because the function $F_{\mathrm{MP}}(E)$ (10) does not change during unimolecular dissociation (1) or (2). Indeed, the decrease of the $A B C$ molecules with the vibrational energy $E$ is exactly compensated for by the increase in the number of the $A B$ molecules with the vibrational energy $E-D_{1}$. Thus, the members related to dissociation turn out to be excluded from the equations for $F_{\mathrm{MP}}(E)$. So the function $F_{\mathrm{MP}}(E)$ turns out to be rather smooth like the one discussed above (7), whereas each of the distribution functions for separate components $f^{A B C}(E), f^{A B}(E)$ and $f^{A}(E)$ may be distorted greatly by dissociation.

To find the yields $\beta$ and $\beta_{A B}$ using the function $F_{\mathrm{MP}}(E)$ one can take advantage of the well-known fact that the unimolecular dissociation rate is highly dependent on the molecular energy $E$. This makes it possible to introduce such boundary energies $E_{D_{1}}$ and $E_{D_{2}}$ that we can consider with a sufficient accuracy such that $A B C$ molecules with their energy $E<F_{2}$ d d not dissociate and all the molecules with $E>E_{D_{1}}$ dissociate i to $A B$ and $C$ (similarly for the $A B$ molecule with its energy $E$ approximating $E_{D_{2}}$ ). If we neglect completely the molecular collisions at all the stages of activation and reaction, we can consider tha $E_{D_{1}}=D_{1}$ and $E_{D_{2}}=D_{1}+D_{2}$. If the energy loss is allowed for, say, in their collisions with a cold buffer, $E_{D_{1}}>D_{1}$. In this case $E_{D_{1}}$ is determined by that critical overexcitation level of the molecule when its dissociation rate equals the rate of cooling. The difference $\left(E_{D_{1}}-D_{1}\right)$ increases as the number of atoms in a molecule increases.

Thus, the values of $\beta$ and $\beta_{A B}$ are determined as

$$
\beta=\int_{E_{D_{1}}}^{\infty} F_{\mathrm{MP}}(E) d E
$$




$$
\beta_{A B}=\int_{E_{D_{1}}}^{E_{D_{2}}} F_{\mathrm{MP}}(E) d E
$$

The exact definition of the composite function $F_{\mathrm{MP}}(E)$ for an arbitrary molecule is, generally speaking, a difficult task. But in our case this is not necessary since for the problem concerned it is only the width of energy distribution function that is of principal importance. Below we shall consider that the distribution width is given by formula (9) for a $s$-multiple degenerate harmonic oscillator. The $s$ value corresponds to the number of vibrational degrees of freedom of the molecule. It must be also stressed that the formula turns out to be valid for an arbitrary system in the following two limiting cases. First, with $\bar{n} \ll s$ expression (9) transforms to the Poisson distribution dispersion $\delta n=\sqrt{\bar{n}}$ which, as known, is formed during MP excitation in the case when opposite induced downward transitions can be neglected. ${ }^{11}$ Secondly, with $\bar{n} \gg s$ it follows from (8) that $\delta n / \bar{n}=1 / \sqrt{s}$ which agrees with the Boltzmann distribution width calculated with the use of the quasi-classical expression for density of states $\rho(E) \sim\left(E+E_{z}\right)^{s}$, where $E_{z}$ is the energy of zero-point vibrations.

In both cases the distribution function $F_{\mathrm{MP}}(E)$ with $\bar{n} \gg 1$ and $s \gg 1$ turns out to be close to the Gaussian one. For definiteness we shall consider the distribution $F_{\mathrm{MP}}(E)$ to be Gaussian in such an arbitrary case, too

$$
F_{\mathrm{MP}}(E)=\frac{1}{\sqrt{2} \pi \delta E} \exp \left[-\left(\frac{\mathrm{E}-\overline{\mathrm{E}}}{\sqrt{2} \delta \mathrm{E}}\right)^{2}\right]
$$

where $\bar{E}$ is the energy stored by a molecule, and the dispersion $\delta E$ is given by expression (9). Now it is possible to write explicit expressions for $\beta$ and $\beta_{A B}$ through the error functions erf $x^{6}$

$$
\begin{gathered}
\beta=\frac{1}{2}\left[1+\operatorname{erf}\left(\frac{\bar{E}-E_{D_{1}}}{\sqrt{2} \delta E}\right)\right] \\
\beta_{A B}=\frac{1}{2}\left[\operatorname{erf}\left(\frac{\bar{E}-E_{D_{1}}}{\sqrt{2} \delta E}\right)-\operatorname{erf}\left(\frac{\bar{E}-E_{D_{2}}}{\sqrt{2} \delta E}\right)\right]
\end{gathered}
$$

The maximum yield of desirable product $\beta_{A B}^{\max }$ is expressed as

$$
\beta_{A B}^{\max }=\operatorname{erf}\left(\frac{E_{D_{2}}-E_{D_{1}}}{2 \sqrt{2} \delta E}\right)
$$

and can be reached with $\bar{E}=E_{D_{1}}+E_{D_{2}}$. 
The fact that the distribution $F_{\mathrm{MP}}(E)$ is narrow, that is, the condition

$$
\delta E \ll E_{D_{2}}-E_{D_{1}}
$$

can be fulfilled, enables us to make use of the asymptotic expansion ${ }^{6}$ of erf $x$ in (13a) and (13b). Let us give an expression for the value $\left(\beta_{A B} / \beta\right)_{1 / 2}$, that is, a relative yield of desirable product with $\beta=\frac{1}{2}$. Such a value of $\beta$ can be obtained with $\bar{E}=E_{D_{1}}$, and in this case

$$
\begin{aligned}
\left(\beta_{A B} / \beta\right)_{1 / 2} & =\operatorname{erf}\left(\frac{E_{D_{2}}-E_{D_{1}}}{\sqrt{2} \delta E}\right) \\
& \simeq 1-\sqrt{\frac{2}{\pi}} \frac{\delta E}{\left(E_{D_{2}}-E_{D_{1}}\right)} \exp \left[-\left(\frac{E_{D_{2}}-E_{D_{1}}}{\sqrt{2} \delta E}\right)^{2}\right]
\end{aligned}
$$

From expression (16) under condition (15) it follows that $\beta_{A B} / \beta \approx 1$. This means that in case of IR MP initiation of reaction effective synthesis of the desirable $A B$ product is possible.

It should be also noted that expressions (13a) and (13b) only approximate the true values $\beta$ and $\beta_{A B}$, rather roughly in some cases. First of all, the values $\beta$ and $\beta_{A B}$ calculated with the use of expressions (11) from composite distribution function (10) are approximated. Besides, the distribution $F_{\mathrm{MP}}(E)$ will be distorted in dissociation since the cross-sections of radiation absorption and quantum state density are different for the $A B C, A B$ and $A$ molecules, with the total energy being the same. All this, however, (and especially the use of expression (12)) does not result in any essential limitations on the conclusion on the possibility of effective synthesis of desirable product during MP excitation. Indeed, of principal importance is oniy the narrowness (condition (15)) of laser-induced energy distribution $F_{\mathrm{MP}}(E)$ which is a characteristic feature of MP excitation. It is because of this that the possibility of effective synthesis of desirable product turns out to be a universal feature of collisionless MP excitation of molecules.

\section{COMPARISON BETWEEN THERMAL AND MP INITIATIONS OF REACTIONS}

The simple model of successive dissociation of molecules considered above enables one to draw an important qualitative conclusion on reducing the number of path-ways of photochemical reaction when 
changing from thermal (equilibrium) to IR MP collisionless (nonequilibrium) initiation.

Let us consider now a specific case of dissociation of the $\left(\mathrm{CF}_{3}\right)_{3} \mathrm{CBr}$ molecule $^{6}(\bar{S}=36)$ and assume $E_{D_{2}}-E_{D_{1}}=20000 \mathrm{~cm}^{-1}$. With $T=$ $1000 \mathrm{~K}$ and equal preexponentials this results in $k_{2} / k_{1}=318$. Figure $1 \mathrm{a}$ presents the calculated dependences of the relative yield of desirable product $\beta_{A B} / \beta$ on the total yield $\beta$ for the cases of thermal initiation (expression (6)) and MP initiation (expression (13)). In case of MP initiation (curve 2) the value of $\beta_{A B} / \beta$ remains close to unity almost up to full conversion of initial substance, that is, $\beta \approx 1$. At the same time in case of thermal initiation (curve 1) the value of $\beta_{A B} / \beta$ tends quickly to zero as $\beta$ increases. Now we are going to compare the values of $\left(\beta_{A B} / \beta\right)_{1 / 2}$ (expressions (6) and (16). At thermal initiation $\left(\beta_{A B} / \beta\right)_{1 / 2}=3 \cdot 10^{-3}$; at MP initiation $\left(\beta_{A B} / \beta\right)_{1 / 2}=0.998$. The comparison of these two values vividly demonstrates the possibility of highly effective directed synthesis at collisionless MP initiation, whereas at thermal initiation such a process is impossible.

For the cause of such a high increase in efficiency of desirable product yield $\beta_{A B} / \beta$ when changing from thermal to MP initiation of reaction to be more vivid, let us consider the vibrational energy distributions for the $A B C, A B$ and $A$ molecules formed in these reactions (Figure 2). As it may be seen from Figures 1a and 1b, the average energy of the $A B$ molecules at MP initiation is much lower (by $\sim D_{1}$ ) than that at thermal initiation. Really, $A B$ molecules are formed (at the instant of dissociation) cold, that is, with their energy

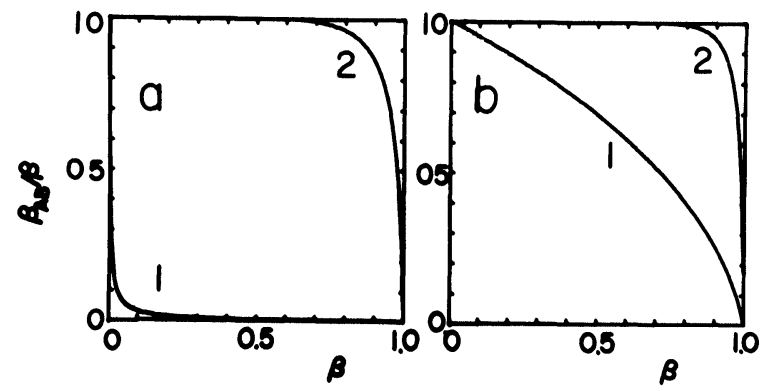

FIGURE 1 Dependence of the relative yield of desirable product $\left(\beta_{A B} / \beta\right)$ on the total yield of reaction at thermal (curves 1) and MP (curves 2) initiation of reaction. (a) $k_{2} / k_{1}=318, \quad E_{D_{2}}-E_{D_{1}}=20000 \mathrm{~cm}^{-1}, S=36$. (b) $k_{2}=k_{1} ; \quad E_{D_{2}}=2 E_{D_{1}}=$ $48000 \mathrm{~cm}^{-1}, S=36$. 

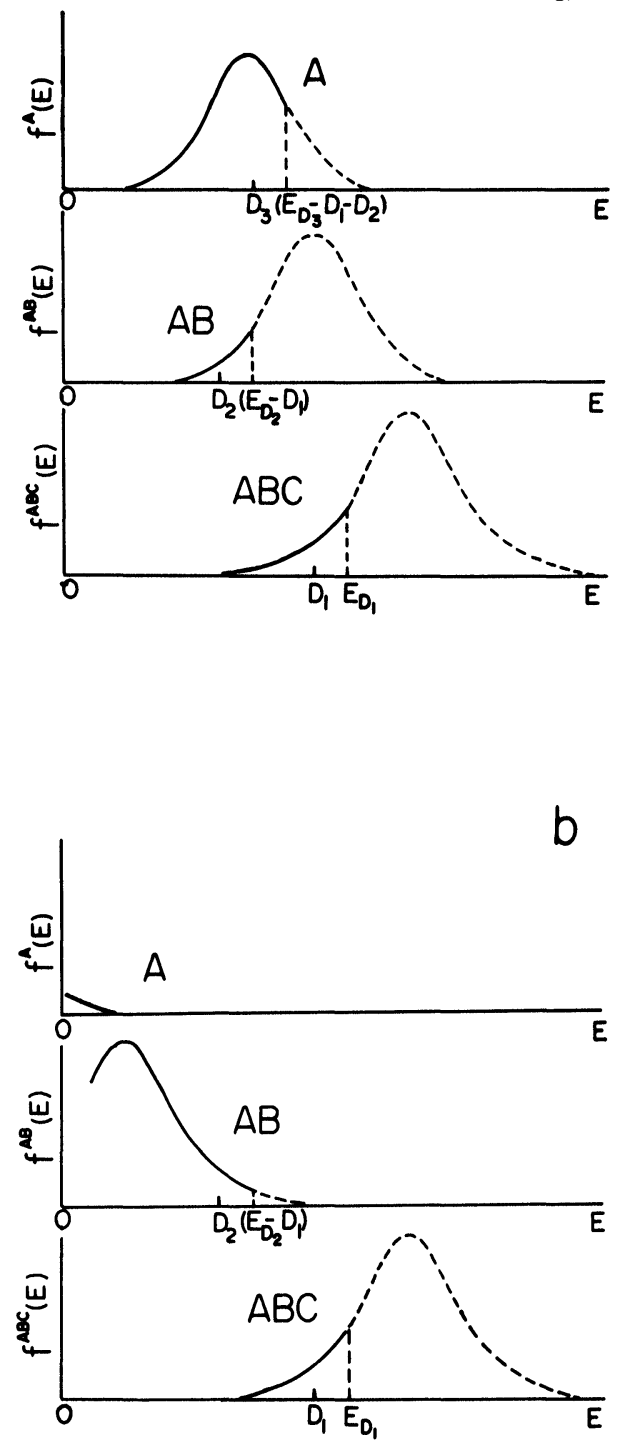

FIGURE 2 Vibrational energy distributions for the initial molecule $A B C$, the desirable product $A B$ and the secondary product $A$. (a) Thermal initiation of reaction. (b) IR MP initiation of reaction. 
being $E_{A B C}-D_{1}$. But in a thermal reaction they quickly gain in collisions some additional energy $\sim D_{1}$. This comes about in times shorter than the dissociation time, and so right after the $A B C$ molecules dissociate the $A B$ product is also "ready" for dissociation (Figure $2 \mathrm{~b}$, curve 2). In this case the vibrational temperatures $T$ of all the components are equal, that is, $T_{A}=T_{A B}=T_{A B C}$. At the same time in case of collisionless MP dissociation the $A B$ molecules remain sufficiently cold, and there will be no dissociation of $A B$ if MP excitation does not go on up to the value $E_{D_{2}}$. If some small part of $A B$ should dissociate (Figure $2 \mathrm{a}$, curve $f^{A B}(E)$ ), the average energy of the $A$ molecules is smaller than that of $A B$ by $\sim D_{2}$. Thus, at MP initiation the relation $T_{A}<T_{A B}<T_{A B C}$ is valid.

\section{CONCLUSION}

Thus, it has been shown herewith that one of the characteristic features of collisionless IR MP dissociation of polyatomic molecules is the possibility of suppressing the successive stages of reaction which bring about considerable fragmentation at thermal dissociation. Reducing the number of reaction pathways, that is, increasing the degree of dissociation directivity, as we pass from thermal dissociation to MP dissociation, results from extreme nonequilibrium of MP initiated reactions and a small width of vibrational energy distribution.

It should be noted that a change from thermal to laser-induced reaction can be observed in experiments as the exciting laser pulse duration or the pressure are decreased. The following reactions of (1)-(2) type were investigated at laser excitation; $\left(\mathrm{CF}_{3}\right)_{3} \mathrm{CBr} \rightarrow\left(\mathrm{CF}_{3}\right)_{3} \mathrm{C} \cdot \rightarrow\left(\mathrm{CF}_{3}\right)_{2} \mathrm{C:}^{6}$ and cyclobutyl acetate $\rightarrow$ cyclobutene $\rightarrow 1,3$-butadiene. ${ }^{12}$ Both papers point to the possibility of effective synthesis of intermediate product when irradiated by rather short pulses. In Ref. 6 the predicted increase of intermediate (desirable) product was observed as the pulse duration was decreased, that is, when passing from collisional to collisionless initiation of dissociation.

In conclusion, we should note the following:

1. Everywhere in the above we considered the case when $k_{2} \gg k_{1}$ that is less favourable for effective formation of the desirable $A B$ product. The result does not change qualitatively with $k_{2}=k_{1}$ since 
the use of MP excitation instead of thermal initiation also increases the efficiency of formation of the desirable product. The results of calculation on the basis of Eq. (4) (curve 1) and expressions (13) (curve 2) are given in Figure $1 \mathrm{~b}$.

2. A greater gain in changing from thermal to MP initiation of reaction can be attained on condition (3), when the dissociation product $A B$ (a molecule or a radical) is thermally less stable than the original product $A B C$. In the reverse case when the desirable $A B$ product is thermally much more stable than the initial $A B C$ product, the use of IR MP initiation of reaction instead of thermal initiation must not cause an essential increase in efficiency of formation of the desirable product.

\section{Acknowledgements}

The authors express their deep appreciation to V. N. Burimov, A. P. Sviridov and A. A. Ishchenko for cooperation and useful discussions.

\section{References}

1. J. G. Calvert and J. N. Pitts, Jr., Photochemistry (John Wiley and Sons, Inc., New York-London-Sydney, 1965).

2. R. V. Ambartzumian and V. S. Letokhov, in: Chemical and Biochemical Applications of Lasers 2, Vol. 3 ed. C. B. Moore (Academic Press, New York-San Francisco-London, 1977).

3. C. D. Cantrell, S. F. Freund and J. L. Lyman, in : Laser Handbook, Vol. III, ed. M. L. Stitch (North-Holland, Amsterdam, 1979).

4. V. S. Letokhov and A. A. Makarov, Advances in Phys. Sci.-Uspekhi Fiz. Nauk. (Russian) 134, 45 (1981).

5. V. N. Bagratashvili, V. S. Letokhov, A. A. Makarov and E. A. Ryabov, Laser Chemistry 1 (1982) and following issues.

6. V. N. Bagratashvili, V. N. Burimov, M. V. Kusmin, V. S. Letokhov and A. P. Sviridov, Laser Chemistry 1 (3), 133 (1983).

7. V. N. Kondratjev and E. E. Nikitin, Kinetics and Mechanism of Gasphase Reactions (Naukas, Moscow, 1974) Russian.

8. M. V. Kus'min (to be published).

9. J. G. Black, P. Kolodner, M. J. Shults, E. Yablonovitch and N. Bloembergen, Phys. Rev. A11, 704 (1979).

10. E. Thiele, J. Stone and M. F. Goodman, Chem. Phys. Lett. 66, 457 (1979).

11. E. S. Medvedev, Chem. Phys. 41, 103 (1979).

12. H. H. Nguyen and W. C. Danen. J. Am. Chem. Soc. 103, 6253 (1981). 